\title{
Le Brésil à la découverte du développement local ${ }^{1}$
}

\author{
André Joyal ${ }^{2}$ et Dante Martinelli ${ }^{3}$
}

Lors d'un séminaire de doctorant sur le développement local présenté en 1993 au Département d'économie de l'Université fédérale de Rio de Janeiro, tous les participants avaient manifesté peu d'enthousiasme envers cette démarche ${ }^{4}$. La forte urbanisation que connaît le Brésil et l'absence de «milieu » susceptibles de donner emprise à une stratégie appuyée sur l'interrelation d'acteurs locaux furent avancées comme explication.

Et pourtant, le taux d'urbanisation se rapproche beaucoup de celui observé au Canada où la pratique du développement local se répand depuis déjà plusieurs années. Le Canada, d'ailleurs, par sa superficie et sa structure administrative fédérale, présente plusieurs similitudes avec le Brésil. En fait, la stratégie économique favorisée par le gouvernement central de Brasilia ne manque pas de points communs avec celle observée au nord du $45^{\mathrm{e}}$ parallèle depuis une cinquantaine d'années. En effet, à l'instar de nombreux pays industrialisés, les années qui ont succédé le second conflit mondial ont été marquées du sceau d'un développement par le haut ayant pour but, com-

\section{Le Canada, par sa superficie et sa structure administrative fédérale, présente plusieurs similitudes avec le Brésil.}

problème endémique de la corruption, la situation se complique encore davantage. S'ajoute à ce tableau l'insatisfaction des besoins primaires ou essentiels. Est-il opportun de chercher à sensibiliser des telles populations à la nécessité de développer une culture entrepreneuriale lorsqu'elles sont aux prises avec des problèmes quotidiens fondamentaux tels la faim, le logement, le transport, etc. ?

En plus de ces problèmes structuraux, le sociologue Ricardo Abramovay ${ }^{5}$ signale deux obstacles majeurs susceptibles d'entraver toute démarche de développement local. Le premier se rapporte aux petites municipalités qui peuvent difficilement être prises comme des unités servant de base à la prospective territoriale à cause d'un vide organisationnel entre ces municipalités et l'État fédéral. Le deuxième concerne les pratiques administratives en vigueur conduisant à sélectionner les municipalités bénéficiaires du Programme national de renforcement de l'agriculture familiale (PRONAF) ou du programme Communauté solidaire. Les critères retenus pour une telle sélection seraient d'une grande pauvreté. Sans une amélioration de ces derniers, on nagerait duction des disparités régionales. Et, toujours à l'instar de ce qui s'observe en Occident, l'inefficacité de ces grands programmes nationaux n'a pas tardé à se manifester. Dans ces conditions comment ne pas envisager la mise en œuvre d'une démarche associée au développement local?

Au Brésil, c'est davantage au Nordeste que la participation des gens au processus démocratique, caractéristique première du développement local, soulève de nombreuses difficultés. En effet, que peut-on attendre d'une population très souvent analphabète et dépendante depuis toujours des pouvoirs supérieurs (gouvernement et grands propriétaires terriens, les trop fameux coroneis)? Quand se pose, en plus, le dans l'arbitraire. En présence d'une telle situation, l'exemple québécois des municipalités régionales de comté et celui de la mise en œuvre de la politique nationale de la ruralité pourraient s'avérer intéressants pour le Brésil

Le présent article, en s'appuyant sur la documentation brésilienne récente et sur l'observation des faits en différentes régions du Brésil, vise à mettre en évidence quelques tendances actuelles des politiques publiques de développement régional et rural. Des résultats partiels d'une étude comparative de deux échantillons de PME en milieu non métropolitain (État de Sao Paulo et Centre-du-Québec) permettra de constater à la fois des similitudes et des distinctions dans le com- 
portement des dirigeants d'entreprise en tant qu'acteurs du développement local.

\section{Renforcement des responsabilités à l'échelon local}

Une meilleure prise en charge (empowerment ou capacity building) des régions en difficulté ne se conçoit autrement qu'en prenant compte des valeurs qui caractérisent les différentes communautés concernées. Ici, Tania Zapata ${ }^{6}$ abonde dans le même sens en parlant de capacitação qui implique des dimensions techniques, pédagogiques et diverses formes d'apprentissage qui favorisent une plus grande autonomie des gens. Cette auteure poursuit en signalant qu'une dynamique de développement local est en voie d'instauration en diverses régions, ce qui confère aux acteurs locaux davantage de possibilités de prise en main de leur destin.

Toutefois, la décentralisation de l'économie ne doit pas être l'occasion pour l'État d'abandonner ses responsabilités en matière économique. Au Canada comme au Brésil, il revient à l'État d'aider les populations locales à s'émanciper de son influence et de celle des grandes entreprises. En somme, l'État doit aider les gens à compter davantage sur eux-mêmes et, pour ce faire, aussi paradoxal que cela puisse paraître, il doit fournir les moyens pour y parvenir. L'un d'entre eux, tout à fait indispensable, se rapporte à l'assistance à un organisme responsable de mettre en branle une stratégie de développement local en assumant les fonctions d'animateur et de dispensateur de l'information liée à l'essor d'initiatives locales de création d'emplois. Pour le Brésil, les SEBRAE estaduais offrent une excellente illustration de la responsabilité d'un tel organisme ${ }^{7}$.

Intervient également le ministère de l'Intégration nationale à travers son secrétariat des programmes régionaux intégrés. Il s'agit de stimuler la participation à l'action sociale en tant que facteur endogène de développement régional. Par la mobilisation des acteurs locaux et de leurs institutions à l'échelle micro ou méso-régionale, on espère susciter un état d'esprit nouveau. L'implantation de forums de développement devrait conduire à la mise en œuvre de projets pilotes de développement méso-régional intégré et soutenable à l'intérieur de pas moins de 14 méso-régions réparties sur l'ensemble du territoires. Dans ces con- ditions, Franklin Coelho ${ }^{8}$ considère que le développement économique local prend la forme d'une construction à l'intérieur d'un milieu productif innovateur où s'expriment diverses coopérations entre les acteurs des réseaux sociaux qui s'y trouvent. Ainsi prend place l'avènement d'un développement humain soutenable.

\section{La décentralisation de l'économie ne doit pas être l'occasion pour l'État d'abandonner ses responsabilités en matière économique.}

Quant à Ricardo Abramovay ${ }^{9}$, il se réfère au développement local en signalant deux présupposés fondamentaux. Le premier se rapporte à la conviction que le seul jeu du marché ne peut suffire à niveler les disparités entre les régions. Le deuxième se rapporte à l'importance de certaines infrastructures pré-requises au développement. Et il poursuit en soulevant la question : « Mais quel est ce local ? » S'agit-il d'un lieu où l'on trouve les ressources requises pour l'amélioration de la qualité de vie ? La réponse à cette interrogation se trouve dans la vaste littérature existant aujourd'hui qui montre que, dans un monde toujours plus globalisé, il ne manque pas de démarches innovatrices mises en place à l'échelon des petites communautés faisant appel à des interrelations individuelles de nature avant tout informelles' On reconnaît l'allusion au fameux dicton « Penser globalement, agir localement ». Ces individus appelés à jouer un rôle clé font partie des forces vives d'un milieu donné.

Il est aussi beaucoup question de capital social dans la documentation brésilienne ces dernières années. L'expression, comme on le sait, se rapporte aux interrelations qu'entretiennent les acteurs locaux à l'intérieur de réseaux formels ou informels dans un lieu donné en s'appuyant sur une confiance réciproque. Juarez de Paula ${ }^{10}$ définit le capital social comme un ensemble de valeurs ou normes informelles particulières aux individus faisant partie d'un groupe et qui leur permettent de coopérer entre eux. En réalité, le capital social serait une notion servant à décrire beaucoup plus que les caractères interrelationnels entretenus par les individus appartenant à une collectivité dûment structurée. En effet, le concept évoque l'intensité d'activités de réseautage associées à la vie économique des forces vives locales sur un espace 
d'intervention servant d'assise au développement local.

Les régions en difficulté seraient en déficit de capital social; or, pour mettre en branle une stratégie susceptible de conduire à des lendemains meilleurs, la cohésion sociale doit imposer sa présence. C'est pourquoi le développement local intégré et soutenable (DLIS) représente un exemple type d'une stratégie prenant son appui sur le capital social. Ainsi, le capital social non seulement sert à promouvoir le développement, mais il peut en plus assurer la soutenabilité. On y trouve un élément favorable à l'émergence de PME.

\section{Quelle place pour les PME?}

Au Canada, le lien entre l'essor des PME et le développement local ne s'observe pas toujours aisément ${ }^{11}$. Ce lien s'observe encore plus difficilement au Brésil. C'est que montre ici quelques résultats tirés d'une étude comparative entre deux échantillons de 60 PME pour lesquels le même questionnaire fut utilisé, soit dans la région de Trois-Rivières, en milieu rural, et dans le Nord-est de l'État de Sao Paulo dans des villes d'importance moyenne. C'est en raison de l'impossibilité de trouver des PME manufacturières dans des villages au Brésil qu'il a fallu procéder différemment en optant pour des villes dont la population varie entre 20000 et 100000 habitants. Il s'agissait de voir, entre autres choses, dans quelle mesure les PME en milieu non métropolitain utilisent des acteurs de leur environnement immédiat comme source d'information en vue d'élaborer leurs diverses stratégies. Pour les PME québécoises, il s'agissait, on le comprendra, d'identifier l'importance du rôle exercé par les sociétés d'aide au développement des collectivités et des centres locaux de développement et toute autre structure d'aide à la création ou à la consolidation d'entreprises locales qui interviennent comme sources d'information aux PME.

\section{Les PME brésiliennes et québécoises}

Le tableau 1 présente les principales caractéristiques des deux échantillons. On trouve des similitudes entre les deux échantillons en ce qui concerne le chiffre d'affaires moyen et l'âge des entreprises.

Par ailleurs, le nombre d'employés moyen des PME brésiliennes est supérieur, même si l'on ne peut tirer de conclusion concernant la représentativité des échantillons. Mais c'est cependant du côté des exportations que l'on enregistrerait la plus grande distinction entre les deux échantillons. On voit que la proportion des ventes allant à l'exportation est plus que trois fois plus élevée que celle des exportatrices brésiliennes.

\section{Tableau 1 - Caractéristiques des PME québécoises et brésiliennes}

\begin{tabular}{lcccc}
\hline PME & \multicolumn{2}{c}{ Québécoises } & \multicolumn{2}{c}{ Brésiliennes } \\
\hline Caractéristiques & Moyenne & Médiane & Moyenne & Médiane \\
\hline Chiffres d'affaires en 1997 (\$ Can) & 4,780 & 2,000 & 4,346 & 1,100 \\
Nombre d'employés & 39 & 21 & 51 & 24 \\
Âge de l'entreprise & 21 & 18 & 22 & 18 \\
\hline Proportion des PME exportatrices & 48,6 & & 16,4 & \\
Pourcentage des ventes pour les exportations & 26,7 & & & \\
\hline Taille de l'échantillon & \multicolumn{3}{c}{60} &
\end{tabular}

L'État de São Paulo étant situé à près de 2000 kilomètres de Buenos Aires, on comprend que l'effet de proximité exercé par les États-Unis sur les entreprises québécoises ne trouve pas son équivalent auprès des entreprises de cet État. Cette faible exportation des entreprises brésiliennes peut créer un sentiment de fausse sécurité, mais risque de coûter cher au Brésil, car celui-ci serait l'un des rares pays de nos jours à pouvoir prospérer sans miser sur l'exportation. En cherchant à conquérir de nouveaux marchés, les entreprises brésiliennes se verraient offrir le meilleur moyen d'apprendre à produire suivant l'évolution de la demande des consommateurs. Et pour y parvenir, les dirigeants brésiliens ont besoin d'innovation de 
diverses natures. Peuvent-ils trouver dans leur environnement immédiat les stimulants susceptibles de les inciter à innover, comme le souhaite tout agent de développement local?

\section{Le recours aux ressources externes}

Le tableau 2 met en évidence la fréquence du recours aux ressources externes qui incluent une gamme va- riée d'acteurs locaux et extérieurs à la région où se trouvent les entreprises.

C'est ici que se fait le lien avec le développement local. Les ressources auxquelles font appel en très grand nombre les entreprises sont également celles qui sont les plus utilisées : les clients, les fournisseurs, les sous-traitants, les consultants et les médias d'information.

\section{Tableau 2 - Fréquence du recours aux ressources externes par les PME}

\begin{tabular}{lccc}
\hline Type de recours & Québécoises $^{*}$ & Brésiliennes $^{*}$ & Signification $^{* *}$ \\
\hline Clients & 6 & 7 & - \\
Fournisseurs & 6 & 7 & 0,002 \\
Sous-traitants & 4 & 5 & - \\
Association sectorielle & 2 & 2 & - \\
Agent de développement & 2 & 0 & 0,000 \\
local (commissaire industriel) & & & \\
Organismes socio-économiques & 1 & 3 & 0,000 \\
Organismes gouvernementaux & 3 & 1 & 0,000 \\
Foires commerciales & 3 & 2 & 0,014 \\
Consultants & 2 & 6 & 0,000 \\
Médias d'information & 5 & 4 & - \\
Centre de recherche & 1 & 1 & - \\
Réseaux d'affaires & 2 & 1 & 0,007 \\
Contacts informels avec d'autres PME & 2 & 5 & - \\
\hline
\end{tabular}

* Codification pour la fréquence d'utilisation : aucun contact $=0$; moins d'une fois par an $=1 ; 1$ fois par an $=2 ; 2$ fois par an $=3 ; 4$ fois par an $=4 ; 1$ fois par mois $=5$; aux 3 semaines $=6$; aux 2 semaines $=7$; au moins une fois par semaine $=8$.

** Le test non paramétrique Mann-Whitney fut utilisé pour produire ce calcul. L'absence de chiffre indique une différence non significative entre les deux échantillons.

Les PME des deux échantillons se distinguent l'une par rapport à l'autre par le type de soutien environnemental privilégié : les foires commerciales, les organismes gouvernementaux, les consultants, les contacts informels et les organismes socio-économiques. Si les PME québécoises recourent peu aux services gouvernementaux, les PME brésiliennes le font encore moins. Et ces dernières ignorent totalement ce que sont les organismes d'appui à l'entreprise (autres que SEBRAE surtout spécialisé en aide à la création d'entreprise) du genre SADC ou CLD, tels qu'ils existent au Québec.

Les autres ressources externes forment trois «coquilles » autour des entreprises pour les deux échan- tillons. La première est évidemment composée des fournisseurs et des clients, suivie d'une seconde moins importante et articulée sur les sous-traitants et les médias d'information. La troisième regroupe l'association sectorielle, les réseaux d'affaires et les centres de recherche. Les PME brésiliennes seraient ainsi, dans l'ensemble, moins avantagées que leurs homologues québécoises par leur environnement immédiat. Et cette situation est encore plus défavorable pour les entreprises brésiliennes non exportatrices. Du côté québécois, les activités de réseautage se rapportent à une proximité organisationnelle plutôt qu'à une proximité géographique. Le fait que bien souvent ces PME représentent l'unique entreprise de leur village oblige à reconnaître l'absence de milieu, tel que conçu par le 
Groupe de recherche européen sur les milieux innovateurs (GREMI).

Pour le Brésil, indépendamment de cette enquête, l'exemple de la petite ville de Pirai (voir la vignette 1) montre bien que la démarche propre au développement local peut, effectivement, conduire à l'implantation de PME innovantes.

\section{Conclusion}

Bien sûr, la création de structures légères, à l'image des SADC canadiennes ou CLD québécois, peu coûteuses et faisant appel à différentes formes de partenariat, présente une avenue prometteuse pour le Brésil. Le pays peut former rapidement les agents de développement nécessaires comme le Québec le fait afin de promouvoir le développement local. Mais on ne change pas en un tour de main les mentalités et les comportements. C'est ce qui exigera du temps pour le Brésil, surtout en ce qui concerne le Nordeste. Il faut avoir à l'esprit la remarque de De Paula ${ }^{12}$ qui signale un obstacle non étranger à la réalité canadienne : la faible crédibilité envers les pouvoirs publics tout échelon confondu : municipal, étatique et fédéral. Les populations locales ont un grand nombre de raisons de mettre en doute la volonté des gouvernements de véritablement s'engager à promouvoir le développement sur la base d'une gestion participative. Ainsi, quand il s'agit de constituer un forum ou un Conseil de développement local, les gens pourront dire : « Encore un de plus... ». Aussi, le défi de réunir des gens à l'intérieur d'un forum ou d'un Conseil de développement local se complique par la présence d'individus appartenant à des forces politiques rivales. On pense ici au problème vécu par les bassins d'emploi en France lorsque les acteurs n'appartiennent pas tous à la même famille politique. Au niveau des petites municipalités, des combats politiques très personnalisés compliquent l'implication des leaders dans un processus de gouvernance locale. Enfin, les élus locaux doivent se mettent en tête le fait que les forums ou conseils de développement local n'existent pas en vue de leur faire ombrage, voire de se substituer à eux.

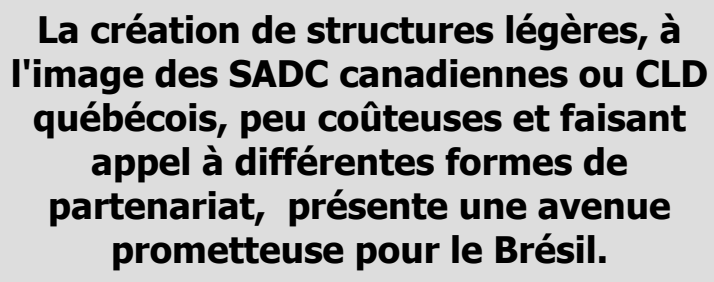

Enfin, pour terminer sur une note optimiste, il peut sembler approprié, pour un pays aussi vaste que le Brésil, de faire allusion à la rupture de l'isolement que favorisent les Nouvelles technologies de l'information et de la communication (NTIC) pour les régions du Nordeste et de l'Amazonie. Les NTIC offrent au développement local des possibilités autrefois inconcevables en favorisant une gestion décentralisée de l'économie nationale. Peut-on y voir une possibilité encore insoupçonnée pour le Nordeste toujours éprouvé par la sécheresse et l'existence de sa multitude de semterra? L'essor des PME innovantes peut-il suffire à fournir aux enfants des sem-terra l'espoir d'un sort meilleur que ne pourrait leur offrir une agriculture de subsistance? Mais encore faut-il que les infrastructures télécommunicationnelles soient conformes aux exigences de la technologie la plus avancée (haut débit), ce qui n'est pas évident, même au Québec. Également, il faut prendre en considération les mises en garde de Polèse et Shearmur ${ }^{13}$ pour qui les NTIC dans les régions périphériques ne répondent pas vraiment au problème de l'isolement. Admettons-le, le développement local, au Brésil comme ailleurs, étant une œuvre de longue haleine, les espoirs sont cependant permis.

\section{Première vignette : Pirai : un exemple de prise en main ${ }^{14}$}

Située à environ 120 kilomètres de Rio de Janeiro, Pirai, une petite ville de 22000 habitants, fut fortement affectée au milieu des années 1990 par les politiques d'ajustements structurels imposées par la Banque mondiale et le FMI. Ainsi, la privatisation d'une entreprise nationale d'électricité a eu pour conséquence le licenciement de 1300 travailleurs, ce à quoi s'est ajoutée la perte de 300 autres emplois à la suite de la restructuration d'une entreprise de papier. C'est alors que le maire (PS) nouvellement élu prit la résolution d'adopter un comportement proactif e $\mathrm{n}$ réunissant différents intervenants du 
milieu en plus de certains fonctionnaires municipaux identifiés comme étant les plus dynamiques. Ce conseil de développement local ainsi formé a permis de dresser un diagnostic de l'état de la situation à partir duquel un plan d'action fut élaboré. Il comprit trois composantes :

a) un programme de promotion du travail et du revenu en milieu rural basé sur la création d'une pisciculture à plusieurs composantes;

b) un programme de création d'emplois et de développement commercial autour de l'implantation d'un parc industriel. Grâce au recours à divers incitatifs de type classique et à une campagne de promotion offrant une vision toute nouvelle de la ville, il fut possible d'attirer rapidement une demi-douzaine de PME innovantes (alimentation, informatique, brasserie, etc.) qui ont su tirer profit de la main-d'œuvre disponible en leur fournissant la formation nécessaire;

c) un programme de création d'emplois sous la forme d'un réseau de coopératives d'artisanat destinées en majorité à des travailleuses, souvent chefs de famille.

L'intégration des milieux rural et urbain et l'ajout d'objectifs sociaux aux objectifs économiques confèrent à la démarche mise de l'avant les caractéristiques principales d'une stratégie de développement local

De toute évidence, cette municipalité ne pouvait pas miser sur le type d'appui qui, à la fin de 2001, est venu au secours de la ville de Chandler en Gaspésie où le gouvernement du Québec a pu préserver 250 emplois en investissant pas moins de... 250 millions de dollars. En 2001, en misant essentiellement sur ses propres moyens, Piraí avait réussi à créer pas moins de 1200 emplois sur son nouveau parc industriel. Évidemment, comme c'est toujours le cas avec le développement local, les acteurs locaux ont fait appel à différents partenaires extérieurs tout en tirant profit de certains programmes gouvernementaux des paliers étatique et fédéral.

\section{Deuxième vignette : PROJETO SEBRAE XINGO}

Il s'agit d'un projet qualifié de développement local intégré et soutenable (DLIS) couvrant quatre États du Nordeste : Alagoas, Bahïa e Pernambuco et Sergipe mis de l'avant par SEBRAE et l'Institut Xingo. Le projet englobe pas moins de 29 municipalités où, à travers la mise en place de forums locaux, on vise la promotion d'une culture entrepreneuriale s'exprimant via l'essor de micro ou de petites entreprises. Ainsi, chacune des municipalités a été invitée à adopter un plan d'action sur la base de priorités dûment identifiées. Un agenda fut déterminé incluant les engagements des acteurs locaux et l'identification des partenaires concernés. Parmi ces derniers, à l'image de ce qui se fait au Canada, on trouve les trois paliers de gouvernement, avec en plus des ONG, des organisations internationales et des entreprises privées locales.

Parmi les priorités mentionnées apparaît la formation de ressources humaines. C'est ce que l'on désigne au Québec comme des interventions de développement de « l'employabilité » destinées à des individus plus ou moins exclus : jeunes ou femmes sans formation académique. L'objectif est de favoriser une meilleure cohésion sociale à la faveur de la mise en valeur du capital social de chacune des municipalités. On ambitionne de modifier les mentalités afin que, dans un futur rapproché, les populations locales soient davantage en mesure de miser sur leurs propres ressources en matière de développement économique.

À titre d'exemple, la municipalité de Nossa Senhora da Gloria, au Sergipe, s'est donné les objectifs suivants : stimuler l'émergence d'une identité locale et la mise en œuvre de nouvelles relations sociales; rechercher et identifier des actions susceptibles d'améliorer le niveau de vie de la population locale; insérer les ressources humaines locales dans le processus de production concurrentiel; préserver les ressources naturelles dans un contexte de développement durable. 


\section{Notes et références}

1 Extrait du livre que les deux auteurs ont fait paraitre récemment: Desenvolvimentolocla o papel das pequenas e medias empressas, Sao Paulo, Manole, 2003, 350 p.

2 André Joyal est professeur d'économie à l'Université du Québec à Trois-Rivières.

3 Dante Martinelli est professeur de gestion à l'Université de Sao Paulo à Ribeirão Preto.

4 André Joyal fut l'invité de ce séminaire placé sous la responsabilité du professeur Henrique Fonseca Netto.

5 Abramovay, Ricardo, 2002, «Obstaculos ao desenvovimento territorial brasileiro », Gazeta Mercantil, 13 mars, p. 2.

6 Zapata, Tania, 1999, «Metodologia de Capaciatação em Apoio ao Desenvolvimento Local », em Desenvolvimento Local no Contexto Urbano, IBASE, Sao Paulo, p. 14.

7 Voir vignette 2 : Projet SEBRAE Xingo.

8 Coelho Franklin, 2001, «Desenvolvimento local e construção social : o território como sujeto ", em Desenvolvi- mento local : dinamicas e estrategias, Rio e Janeiro, Rede DLIS, p. 62.

9 Abramovay, Ricardo, 1998, A Formaçao de Capital Social para o Desenvolviment o local Integrado e Sus-tentavel, II Forum CONTAG de Cooperaçai Ténica,San Luis, Dezembro polycopiado.

10 De Paula, Juarez, 2001, « Desenvolvimento \& gestão compartilhada », em Desenvolvimento local : dinamicas e estatategias, op.. cit.p 145.

11 Joyal, André et Laurent Deshaiens, 1996, «Développement local et PME québécoises innovantes: un lien à explorer », Revue canadienne des sciences régionales, vol. XIX, n 3 , automne, p. 333-348.

12 De Paula, Op. cit.

13 Polese, Mario et Richard Shearmur, 2002, La périphérie face à l'économie du savoir, Montréal, INRS, Université du Québec.

14 Informations recueillies à grâce à trois visites sur place d'André Joyal. 


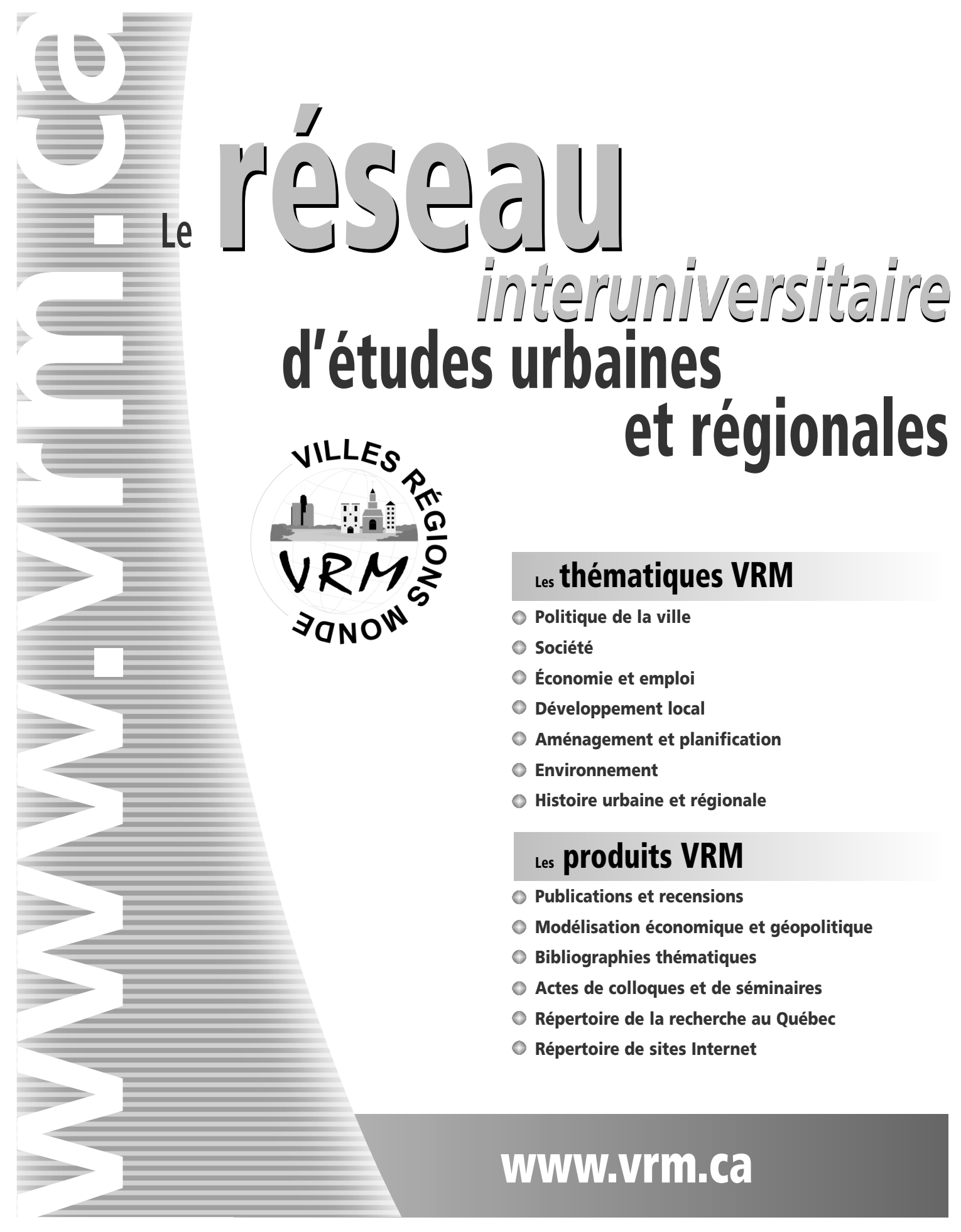

